\title{
ROTATING PROTOPLANETARY GAS DISK IN GG-TAU
}

\author{
Yoshimi KITAMURA ${ }^{1}$, Ryohei KAWABE ${ }^{2}$, Toshihiro OMODAKA ${ }^{3}$, \\ Masato ISHIGURO ${ }^{2}$, and Syoken MIYAMA ${ }^{4}$

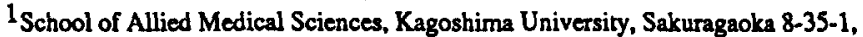 \\ Kagoshima 890, Japan \\ 2 Nobeyama Radio Observatory, Nobeyama, Minamimaki-mura, Minamisaku-gun, \\ Nagano 384-13, Japan \\ ${ }^{3}$ College of Liberal Arts, Kagoshima Univ., Korimoto 1-21-30, Kagoshima 890, Japan \\ ${ }^{4}$ National Astronomical Observatory, Osawa 2-21-1, Mitaka, Tokyo 181, Japan
}

\begin{abstract}
A rotating gas disk was discovered around a T-Tauri star, GG-Tau, in Taurus by using the Nobeyama Millimeter Array (NMA) (Kawabe et al. 1993). To analyze the observed data, we have numerically calculated velocity profiles of $\mathrm{CO}$ emitted from protoplanetary gas disks by taking account of the line radiative transfer.
\end{abstract}

It is generally believed that the solar system was born from a disk of gas and dust, having a radius of $\sim 40 \mathrm{AU}$ and encircling the Sun about five billion years ago (e.g., Hayashi, Nakazawa, and Nakagawa 1985; Cameron 1985). Millimeter interferometric observations of HL-Tau, T-Tau, and DG-Tau, which are T-Tauri stars similar to the Sun, revealed gas disks with radii of a few thousands $A U$ around the stars (e.g., Sargent and Beckwith 1987, 1991). Recently, Skrutskie et al. (1993) detected the ${ }^{12} \operatorname{CO}(J=1-0)$ emission toward GG-Tau using the Nobeyama $45 \mathrm{~m}$ telescope with a spatial resolution of $17 "(2400 \mathrm{AU}$ at the distance to GG-Tau, $140 \mathrm{pc})$. The velocity profile of the CO emission strongly suggests the existence of a rotating gas disk around GG-Tau.

To investigate the distribution and kinematics of the molecular gas found toward GG-Tau, we made aperture synthesis ${ }^{12} \mathrm{CO}(J=1-0)$ observations using NMA on April 2 and May 2 - 4 in 1992 (Kawabe et al. 1993). The size of the synthesized beam was 10" $\times$ 6" (P.A. $\left.=138^{\circ}\right)$ and the velocity resolution was $0.81 \mathrm{~km} \cdot \mathrm{s}^{-1}$.

We have discovered a rotating gas disk around GG-Tau, and have derived several parameters of the gas disk as follows: The radius $R_{\text {disk }}$ is larger than $500 \mathrm{AU}$; the inclination angle $i$ is $\sim 60^{\circ}$; the rotation velocity, $0.8 \mathrm{~km} \cdot \mathrm{s}^{-1} / \mathrm{sin} i$ at $500 \mathrm{AU}$, agrees with Kepler rotation around GG-Tau having a mass of $0.65 M_{\odot}$; the gas kinetic temperature $T$ is $\sim 10 \mathrm{~K}$ at $500 \mathrm{AU}$; and the estimated mass of the disk, $M_{\text {disk }}$ ranges from $1 \times 10^{-4} M_{\odot}$ (from total CO flux) to $0.4 M_{\odot}$ (from kinematical consideration).

For the analysis of our observed data, we calculate velocity profiles of the ${ }^{12} \mathrm{CO}$ $(J=1-0)$ line emitted from protoplanetary gas disks around young stars by taking the line radiative transfer into account (Omodaka, Kitamura, and Kawazoe 1992), based on the Kyoto model (Hayashi, Nakazawa, and Nakagawa 1985). The antenna temperature $T_{\mathrm{A}}{ }^{*}$ of the $\mathrm{CO}$ emission from a disk at a frequency $v$ is calculated by the following equation:

$$
T_{\mathrm{A}}{ }^{*}=\frac{\ln 16 \lambda^{2}}{2 \pi k_{\mathrm{B}}} \frac{1}{H P B W^{2}} \frac{1}{D^{2}} \int_{\text {disk }} P_{v}(\theta, \phi) \kappa_{v} B_{\Downarrow}(T) e^{-\tau_{v}} d V,
$$

where $\lambda$ is the wavelength of the emission, $k_{\mathrm{B}}$ is the Boltzmann constant, HPBW is the beam size of a telescope, $D(=140 \mathrm{pc})$ is the distance to the source, $P_{\mathrm{V}}(\theta, \phi)$ is the beam pattern of a radio telescope, $\kappa_{V}$ is the absorption coefficient for the emission, $B_{V}(T)$ is the Planck function with a temperature $T$, and $\tau_{v}$ is the optical depth along the line of sight. In this equation we assume the LTE condition for the CO emission. We adopt $9 \times 10^{-5}$ as the abundance ratio of the ${ }^{12} \mathrm{CO}$ to $\mathrm{H}_{2}$ molecules, and neglect the dust opacity.

First, we tried model fitting to the ${ }^{12} \mathrm{CO}$ profile detected toward GG-Tau by Skrutskie et al. (1993) with the $45 \mathrm{~m}$ telescope. In our model fitting, it was assumed that 
the mass of GG-Tau is $0.65 M_{\odot}$, and that the gas temperature varies as $13(r / 500 \mathrm{AU})^{-0.5}$ $\mathrm{K}$. Both the radius $R_{\text {disk }}$ and inclination angle $i$ of the disk were changed. The best fit result was obtained for $R_{\text {disk }}=900 \mathrm{AU}$ and $i=67^{\circ}$, as shown by thin solid line in Fig. 1 .

Next, we converted the calculated profile shown in Fig. 1 into the profile for the case of observations by NMA. The profile indicated by the broken line in Fig. 2 shows the converted profile. Furthermore, we changed the velocity resolution into $0.81 \mathrm{~km} \cdot \mathrm{s}^{-1}$, and the corresponding profile is indicated by thin solid line. On the redshifted side, the observed profile agrees with our calculated profile within the uncertainty of the flux scale. On the blueshifted side, however, the observed profile is twice as high as the calculated one. This large difference may be explained by the following model: The disk is considered to be asymmetrical as observed by NMA, and the radii of the disk on the east and west sides are estimated to be $\sim 2000$ and $1000 \mathrm{AU}$, respectively (see Fig. 3). Since the beam size of the $45 \mathrm{~m}$ telescope is $\sim 2000 \mathrm{AU}$, the single dish could not cover the entire disk, and only the redshifted part was covered. As a result, the observed velocity profile of $\mathrm{CO}$ becomes symmetric and shows the double-peaked feature (see Fig. 1). On the other hand, the field of view of NMA completely covered the disk, and the resultant velocity profile has an excess on the blueshifted side (see Fig. 2).

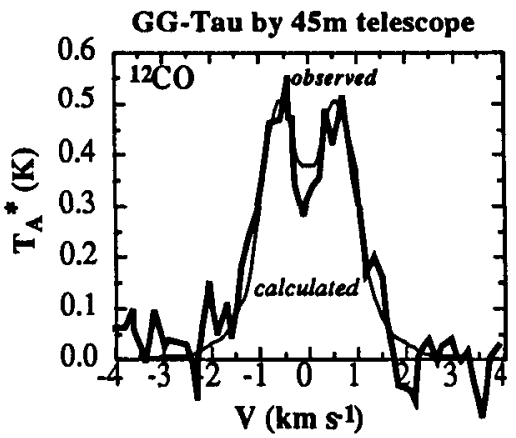

Figure 1. ${ }^{12} \mathrm{CO}(J=1-0)$ profile detected toward GG-Tau by the Nobeyama $45 \mathrm{~m}$ telescope (thick solid line) and a calculated profile (thin solid

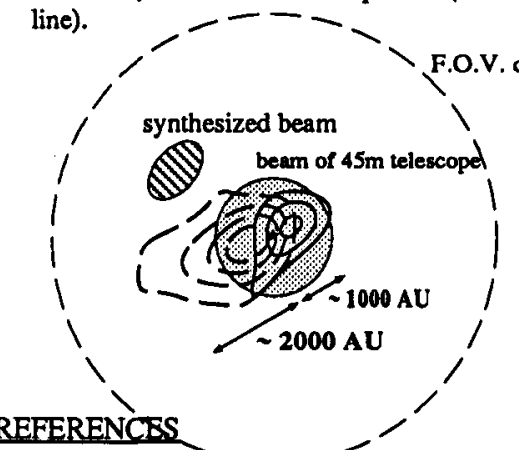

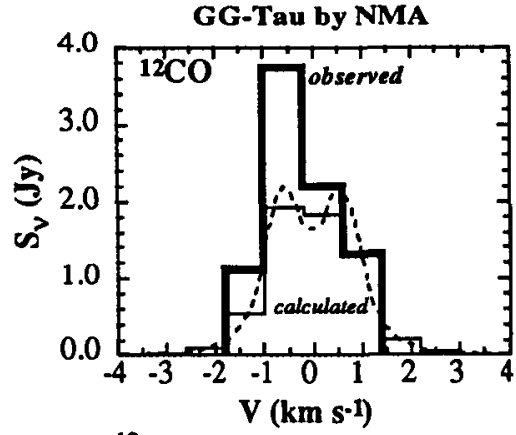

Figure 2. ${ }^{12} \mathrm{CO}(J=1-0)$ profile observed by NMA (thick solid line) and a calculated profile (thin solid and broken lines).

Cameron 1985, in Protostars \& Planets II (Univ. of Arizona Press), p.1073

Hayashi, Nakazawa, and Nakagawa 1985, in Protostars \& Planets II (Univ. of Arizona), p.1100

Kawabe et al. 1993, Ap. J. Letters, 404, L63

Omodaka, Kitamura, and Kawazoe 1992, Ap. J. Letters, 396, L87

Sargent and Beckwith 1987, Ap. J., 323, 294

Sargent and Beckwith 1991, Ap. J. Letters, 382, L31

Skrutskie et al. 1993, Ap. J., 409, 422 